\title{
Design of a Low-Power Wireless Structural Monitoring System for Collaborative Computational Algorithms
}

\author{
Yang Wang *a, Jerome P. Lynch ${ }^{\mathrm{b}}$, Kincho H. Law ${ }^{\mathrm{a}}$ \\ ${ }^{a}$ Dept. of Civil and Environmental Engineering, Stanford University, Stanford, CA 94305 \\ ${ }^{\mathrm{b}}$ Dept. of Civil and Environmental Engineering, University of Michigan, Ann Arbor, MI 48109
}

\begin{abstract}
In this study, a new wireless sensing unit for operation within an automated Structural Health Monitoring (SHM) system is proposed, designed and validated. The design of the wireless sensing unit emphasizes minimization of its power consumption characteristics to ensure it is suited for long-term field deployment in civil structures. The wireless modem integrated with the unit has a long communication range that permits wireless sensors to be spaced over $100 \mathrm{~m}$ apart. A multi-channel high-resolution analog-to-digital converter is included within each sensing unit to provide flexibility for high-fidelity data collection. A key feature of the wireless sensing unit design is the inclusion of a sophisticated computing core that is capable of locally executing engineering algorithms in real-time. As part of the embedded software, a novel communication protocol is written that can accomplish low-latency communications for accurate time synchronization between spatially distributed wireless sensors. To illustrate the capabilities of the wireless monitoring platform, including the execution of extensive computational tasks, a prototype system is fabricated and tested in the laboratory and field. As part of validating the system performance in the field, the vertical acceleration response of the Geumdang Bridge under traffic loading is measured by 14 wireless sensing unit prototypes.
\end{abstract}

Keywords: Wireless structural monitoring, wireless sensor networks, structural health monitoring, modal testing

\section{INTRODUCTION}

Civil structures often experience deterioration and damage as a direct result of extreme loading and hazardous events. For instance, it was reported by the National Bridge Inspection Program (NBIP) that nearly 60,000 highway bridges within the United States are structurally deficient; in other words, their load ratings were well below established safety standards ${ }^{1}$. To protect the public from unsafe structures, current federal requirements necessitate local transportation authorities to visually inspect the entire inventory of highway bridges biannually (there are well over 580,000 highway bridges in the United States). An inherent drawback of visual inspections is that they only consider damage that is visible on the surface of the structure; damage located below the surface would remain elusive to the inspector. With visual inspections both costly and labor intensive, low cost sensing systems that can quantitatively assess the integrity and remaining life of a structure are needed. Often referred to as Structural Health Monitoring (SHM) systems, these systems can augment or potentially replace visual inspections since their desired functionality is to automatically predict, identify, and locate the onset of structural damage ${ }^{2}$.

Autonomous SHM systems employ smart sensor technologies to assist in identifying subtle structural distress based on measured structural response parameters ${ }^{3}$. A core element of the SHM system is the numerical algorithms that are used to screen response data for indications of damage. Various types of structural sensors, including accelerometers, linear variable displacement transducers, strain gages, thermometers, and humidity sensors, can be deployed to provide structural behavioral data used within the SHM process. An important fundamental element of all SHM systems is the data acquisition (DAQ) system that is used to collect and store sensor measurements in a centralized location. Current commercial DAQ systems designed for permanent installation in civil structures are often adaptations of laboratorybased systems that employ coaxial wires between sensors and the data repository. By running cables between sensors and a central data server within a structure, traditional tethered DAQ systems suffer from high installation costs in both time and money. With respect to the installation time, installing extensive lengths of cables can consume over $75 \%$ of the total system installation time ${ }^{4}$. It has been reported that the cost of installing a structural monitoring system can

*wyang98@stanford.edu; phone 1650 723-6213; fax 1650 725-9755; http://eil.stanford.edu/WiMMS/ 
exceed a few thousand dollars per sensing channel ${ }^{5}$. When considering the large number of bridges to be monitored in U.S., the cost of installing tethered structural monitoring systems on all of the nation's bridges would be tremendous.

Recent developments in the fields of microelectromechanical systems (MEMS) and wireless communications have presented exciting new opportunities to reduce the installation costs of structural monitoring systems. MEMS technology helps to reduce the size, power consumption, and cost of structural sensors while wireless technology allow for the collection of sensor measurements without the need for cables. The use of wireless communications as a means of eradicating cables within a structural monitoring system was first illustrated by Straser and Kiremidjian ${ }^{4}$. Their seminal work demonstrated the functionality and cost-effectiveness of wirelessly monitoring civil structures. Lynch et $a l .{ }^{6}$ have advanced wireless SHM by embedding damage identification algorithms directly into wireless sensing units. Their work harnessed the computational resources of wireless sensing units to execute data interrogation algorithms as a means of reducing and simplifying wireless communications within the SHM system. Kottapalli et al. ${ }^{7}$ proposed a two-tiered wireless sensor network topology that especially addresses the power consumption, data rate, and communication range limitations of current wireless monitoring systems. In addition to these efforts, several other research teams have been actively developing an impressive collection of wireless sensing platforms for structural health monitoring ${ }^{3,8-11}$.

Compared to traditional wire-based systems, wireless structural monitoring systems have a unique set of technical challenges. First, wireless sensing units will likely require batteries that have a limited supply of energy. This restriction on the life expectancy of the unit requires advanced local data processing since the wireless radio often consumes the greatest amount of energy than any of the other components in the wireless sensing unit ${ }^{6}$. Second, the transmission of data in a wireless network is inherently less reliable than in tethered networks; reliability reduces as the communication range gets longer. Third, the limited amount of wireless bandwidth usually impedes high-speed realtime data collection from multiple sensors. Fourth, the collection of data from different wireless sensing units may experience variable time-delays because of sensor blockage or clock imprecision in the units ${ }^{12}$. These time delays can negatively impact the accuracy of the numerical procedures executed by the SHM system.

To better address the above challenges, a new wireless structural monitoring system is proposed in this paper. Some of the major design objectives for this new wireless SHM system are: 1) low power consumption, 2) long communication ranges with robust communication protocols for reliable data acquisition, 3) accurate synchronized wireless data collection from multiple heterogeneous analog sensors at an acceptable sampling frequency, 4) high-precision analog-todigital conversion, 5) considerable local data processing capability at the wireless sensing units (to save precious battery energy and to enhance system scalability), and 6) accommodation of peer-to-peer communication among wireless sensing units for collaborative decentralized data analysis. Hardware and software realization of these design objectives are presented in detail within this paper. After the wireless sensing system is designed and constructed, an extensive amount of laboratory and field testing is conducted to validate the system performance within the complex environment posed by civil structures.

\section{COMPONENT-LEVEL DESIGN OF A WIRELESS SENSING UNIT}

A wireless SHM system with a star-topology includes multiple wireless sensing units assembled in a network with one central server coordinating the activity of the network. In our prototype system, the central server can be any type of laptop or desktop computer with a compatible wireless transceiver connected through a typical RS232 serial communication port. Through the wireless transceiver, the central server can communicate with all of the wireless sensing units that have been dispersed throughout the structure. The wireless sensing units are primarily responsible for acquiring sensor measurements, analyzing data, and transferring data to the central server for permanent storage. Therefore, the functional properties of the global SHM system largely depend on the hardware design of the individual wireless sensing units. As discussed in the previous section, some of the key issues considered in the hardware design of the wireless sensing units include restricted power consumption, long inter-nodal distances, and limited local data processing authority. These issues have been especially addressed in the hardware design of the new wireless SHM system proposed in this study. 


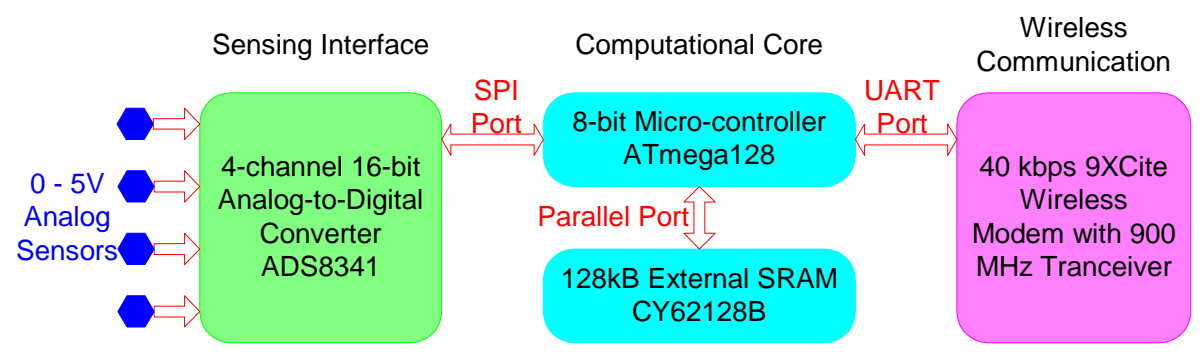

Figure 1. Functional diagram detailing the hardware design of a wireless sensing unit for structural monitoring

A functional diagram of the proposed wireless sensing unit is illustrated in Figure 1. The design of the wireless sensing unit consists of three functional modules: the sensing interface, the computational core, and the wireless communication channel. The sensing interface converts analog sensor signals into a digital format understood by the computational core. The sensing interface consists of the Texas Instruments ADS8341 4-channel 16-bit analog-to-digital converter that can sample data at rates in excess of $100 \mathrm{kHz}$. The digitized sensor data is then transferred to the computational core through a high-speed Serial Peripheral Interface (SPI) port. The core is designed around the low-power 8-bit Atmel ATmega128 microcontroller. In addition to the ATmega128, external Static Random Access Memory (SRAM) is integrated with the computational core to accommodate local data storage and analysis. Through a Universal Asynchronous Receiver and Transmitter (UART) interface, the computational core communicates with the Maxstream 9XCite wireless modem thereby providing a wireless connection between the unit and other devices in the wireless network. The 9XCite operates on the unlicensed $900 \mathrm{MHz}$ radio spectrum and can achieve communication ranges of $300 \mathrm{~m}$ in open space. The reader is referred to a previous paper that provides a more detailed description of the hardware design of the wireless sensing unit ${ }^{13}$.

\subsection{Power consumption}

Restricted power consumption is one of the most important issues to consider when selecting the hardware elements of a wireless sensing unit. While power consumption of each hardware component should be minimized, it must not be done at the expense of the wireless sensing unit functionality. The power consumed by the wireless sensing unit is a function of the voltage and the amount of electrical current supplied to each component. All of the hardware components are internally referenced at $5 \mathrm{~V}$. As shown in Table 1, the active and standby electrical current for each component of the wireless sensing unit is listed. When the wireless sensing unit is active, it is collecting, interrogating or wirelessly transmitting sensor data. In contrast, the wireless sensing unit can be placed in a sleep state from which it can be easily awakened; when sleeping, the unit consumes a minimal amount of electrical current (as denoted by the standby current).

Table 1. Approximate current consumption of the wireless sensing unit

\begin{tabular}{|l|c|c|}
\hline & Active Current & Standby Current \\
\hline Analog-to-digital converter (at 100 Hz) & $1 \mathrm{~mA}$ & $1 \mu \mathrm{A}$ \\
\hline Micro-controller Atmega128 (at 8MHz) & $15 \mathrm{~mA}$ & $40 \mu \mathrm{A}$ \\
\hline SRAM CY62128B & $15 \mathrm{~mA}$ & $15 \mu \mathrm{A}$ \\
\hline Wireless modem 9XCite & $45 \mathrm{~mA}$ & $20 \mu \mathrm{A}$ \\
\hline Support electronics & $1 \mathrm{~mA}$ & $24 \mu \mathrm{A}$ \\
\hline \hline Complete wireless sensing unit & $\mathbf{7 7 m A}$ & $\mathbf{1 0 0} \mu \mathrm{A}$ \\
\hline
\end{tabular}

It should be noted that the wireless radio consumes the greatest amount of electrical power when active $(45 \mathrm{~mA} * 5 \mathrm{~V}=$ $225 \mathrm{~mW}$ ). This finding emphasizes the importance of minimizing the use of the wireless communication channel as a means of preserving battery energy ${ }^{6}$. The total active current of the wireless sensing unit prototype, as measured by a digital multi-meter in the laboratory, is found to be $77 \mathrm{~mA}$ while the standby current is measured as $100 \mu \mathrm{A}$. The wireless sensing unit can operate on any power source providing at least $5.2 \mathrm{~V}$ of voltage potential but in the current prototype construction five ordinary lithium AA batteries (Energizer L91), providing a total voltage of 7.5V, are used. The total life expectancy of the wireless sensing unit using this set of batteries can be calculated. The total energy 
capacity of a single L91 AA battery is over 2900mAh. Therefore, the fully-active continuous life expectancy of the wireless sensing unit is estimated to be:

$$
T_{\text {active }}=\frac{E_{\text {battery }}}{I_{\text {active }}}=\frac{2900 \mathrm{mAh}}{77 \mathrm{~mA}}=37.7 \mathrm{hrs}=1.57 \text { days }
$$

This expected active life is conservative because this assumes the unit is in continuous operation. However, duty cycle usage of the battery allows for the internal cell chemistries to attain equilibrium thereby extending the batteries life expectancy. The standby life-time of the wireless sensing unit is:

$$
T_{\text {standby }}=\frac{E_{\text {battery }}}{I_{\text {standby }}}=\frac{2900 \mathrm{mAh}}{100 \mu \mathrm{A}}=29000 \mathrm{hrs}=1208.3 \text { days }
$$

In all likelihood, wireless sensing units serving as part of a comprehensive structural health monitoring system would be operated on a duty-cycle schedule. For example, units might be programmed to turn on every day for 10 minutes to measure the ambient response of the instrumented structure. If it is assumed that on each day, the system is fully active for 10 minutes of data collection, then the total operation time of the wireless sensing unit is:

$$
T_{10 \text { min-active-per-day }}=\frac{E_{\text {battery }}}{E_{0.167 \mathrm{hr} \text {-active-per-day }}+E_{23.83 \mathrm{hr} \text {-standby-per-day }}}=\frac{2900 \mathrm{mAh}}{77 \mathrm{~mA} \times 0.167 \mathrm{hr} / \text { day }+100 \mu \mathrm{A} \times 23.83 \mathrm{hr} / \text { day }}=190 \mathrm{days}
$$

If the units are only used for 5 minutes every day, then the life expectancy of the unit is calculated to be 330 days.

\subsection{Wireless Communication}

For application within the complex environment posed by civil structures, robust wireless communication architectures are needed. In particular, the anticipated inter-nodal distances could be upwards of $100 \mathrm{~m}$ thereby necessitating a wireless radio with exceptional communication range. However, long communication ranges usually require higher power consumption on the part of the wireless radio; this conflicts with the requirement to reduce power consumption of the wireless sensing unit. In this research, the Maxstream 9XCite wireless modem is selected for the wireless sensing unit because of its capability for relatively long range communication yet only consuming a modest amount of battery energy. The key performance characteristics of the 9XCite wireless modem are summarized in Table 2. As specified, the $90 \mathrm{~m}$ indoor communication range of the 9XCite wireless modem satisfies applications in small and medium-sized civil structures. The raw data transfer rate of the modem is $38.4 \mathrm{kbps}$, but after a robust communication protocol consisting of reliable retry-acknowledgement procedures has been implemented, the effective data transfer rate is approximately $26 \mathrm{kpbs}$. Based on the communication protocol and the subsequent effective data rate, if the sampling frequency of each sensor channel is $50 \mathrm{~Hz}$, then one central server will be able to collect raw data from up to 24 sensing channels sampled continuously in real-time, without the possibility of losing data. The peer-to-peer communication capability of the wireless modem also makes it possible for the wireless sensing units to communicate with each other, thus supporting collaborative local data analysis.

Table 2. Key characteristics of Maxstream 9XCite wireless modem

\begin{tabular}{|l||l|}
\hline Communication Range & Up to 300’ (90m) indoor, 1000’ (300m) outdoor \\
\hline Data Transfer Rate & $38.4 \mathrm{kbps}$ \\
\hline Operating Frequency & $902-928 \mathrm{MHz}$ \\
\hline Channel Mode & $\begin{array}{l}7 \text { channels at Frequency Hopping Spreading Spectrum } \\
\text { (FHSS) mode, or 25 channels at Single Frequency mode }\end{array}$ \\
\hline Supply Voltage & 2.85VDC to 5.50VDC \\
\hline Power Consumption & 55mA transmitting, 35mA receiving, $20 \mu \mathrm{A}$ standby \\
\hline Module Size & 1.6” x 2.825” x 0.35” $(4.06 \mathrm{~cm}$ x 7.17 cm x 0.89 cm) \\
\hline Network Topology & Point-to-point, point-to-multipoint \\
\hline
\end{tabular}

\subsection{Data Collection}

The wireless sensing unit represents an autonomous node within the wireless monitoring system. To each wireless sensing unit, up to four analog sensors with outputs ranging from 0 to $5 \mathrm{~V}$ can be attached at one time. At the core of 

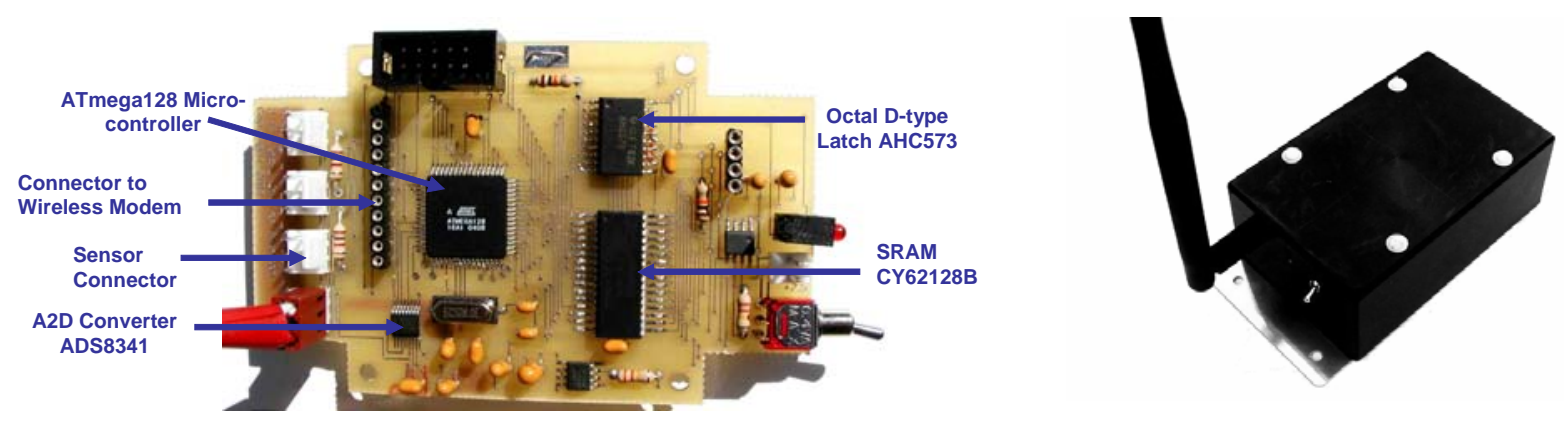

Figure 2. (Left) final printed circuit board and (right) fully assembled wireless sensing unit prototype

the sensing interface is the Texas Instrument ADS8341 four-channel 16-bit analog-to-digital (A/D) converter that can sample sensor outputs at rates as high as $100 \mathrm{kHz}$. Real-time operation of the sensing interface is performed by the embedded operating system through the use of timer interrupt functions native to the ATmega128 microcontroller.

\subsection{Local Data Processing}

ATmega128 8-bit microcontroller is selected to serve as the computational core of the wireless sensing unit. The microcontroller communicates and coordinates with the various functional modules of the wireless sensing unit by running an embedded multi-threaded operating system that resides in its non-volatile flash memory. With an additional $128 \mathrm{kB}$ of external SRAM memory (CY62128B) included in the core, the microcontroller has an impressive capacity for local data storage and analysis. Local data storage is instrumental for real-time wireless data acquisition from multiple sensing units since the central server will collect data from multiple wireless sensing units on a one-by-one basis.

To coordinate the behavior of the wireless sensing unit, an embedded operating system is written for the prototype unit. An attractive feature of the operating system is that it can execute two tasks at one time (multi-threaded tasking). In particular, the unit is capable of collecting data from the sensing interface simultaneous to performing other operational tasks such as transferring data over the wireless modem. The operating system can multitask because of the use of interrupt functions and a very short conversion time $(10 \mu \mathrm{s})$ of the A/D converter. The interrupt functions are necessary to momentarily pause other tasks (such as communications) when data is available for pick-up at the sensing interface. The operating system abstracts the implementation details of the unit hardware for upper layers of engineering analysis software.

\subsection{Fully Assembled Wireless Sensing Unit Prototype}

To ensure the wireless sensing unit is compact, a simple two-layer printed circuit board (PCB) is designed and fabricated. A picture of the completed PCB with integrated circuit chips attached is shown in Figure 2. The final dimension of the PCB is roughly $9.7 \mathrm{~cm}$ by $5.8 \mathrm{~cm}$. To protect the electronics from the harsh weather conditions common to outdoor structural applications (such as installation on long-span bridges), the PCB, wireless radio and batteries are stored within a weather proof plastic container shown in Figure 2. The final dimensions of the wireless sensing unit are $10.2 \mathrm{~cm}$ by $6.5 \mathrm{~cm}$ by $4.0 \mathrm{~cm}$.

\section{SOFTWARE DESIGN FOR THE WIRELESS SHM SYSTEM}

A simple star-topology data acquisition system is designed and implemented. The prototype system includes one central server and multiple wireless sensing units. The central server is responsible for commanding all of the wireless sensing units to collect data, for receiving data from the wireless network, and for storing measurement data in a file server. A desktop or laptop computer connected with a MaxStream 9XCite wireless modem can be used as the central server. Software written for the wireless structural monitoring system is delineated into two parts: software for the central server and embedded software for the wireless sensing units. Since the central server and the wireless sensing units must interface with one another, portions of their software design must be done in tandem. To assist in this effort, a detailed program flow for either the central server or the wireless sensing unit is done using a state diagram approach. The intention of the state diagram is to encode a sequence of actions for both the central server and the wireless sensing 
units given potential scenarios (or states) that correspond to problems typically encountered in an unreliable wireless channel. In the star topology, the central server is assigned the responsibility of ensuring reliable wireless communication. In other words, the central server is playing an "active" role in the communication while the wireless sensing unit plays more of a "passive" role. For example, communication is always initiated by the central server. After the central server sends a command to the wireless sensing unit, if the server doesn't receive an expected response from the unit, the server will resend the last command again until the expected response is received. However, after a wireless sensing unit sends a message to the central server, the unit does not care if the message has arrived at the central server correctly or not because the communication reliability is guaranteed by the server. The wireless sensing unit becomes aware of the lost data only when the central server queries the unit for the same data again. This section describes in detail, the nature of the communication between the central server and the wireless sensing units.

\subsection{Data Synchronization}

For many damage identification and system identification procedures, measurement data individually collected by different sensors must be time synchronized. For example, many modal analysis algorithms require synchronized acceleration data from different nodes of the structure. The design of the communication protocol adopted between the central server and the wireless sensing units to accomplish clock synchronization is shown in Figure 3.

An attractive feature of the 9XCite wireless modem is that it supports point-to-multipoint communications. Using this feature, a broadcasted beacon signal from the central data server's wireless modem is sent to all the wireless sensing units. Once the wireless sensing units receive the beacon signal from the central server, each unit starts data sampling at the requested sampling frequency. This approach of using a beacon signal for synchronization is similar to the approaches employed in other wireless protocols (e.g. 802.11 and 802.15.4). Each wireless sensing unit may not receive the beacon notification from the central server at precisely the same time; some units may start collecting data slightly earlier while others may start later. Because the propagation of the wireless radio frequency (RF) signal takes only nano-seconds in this application, the synchronization error mainly originates from the difference in the time it takes each wireless sensing unit to interpret the broadcasted beacon signal. Using precise timers in the laboratory, the synchronization of multiple sensing units is measured to be within $20 \mu$ s of one another. However, it should be noted that although the system synchronization error is around $20 \mu$ s at the beginning of the data collection, the synchronization

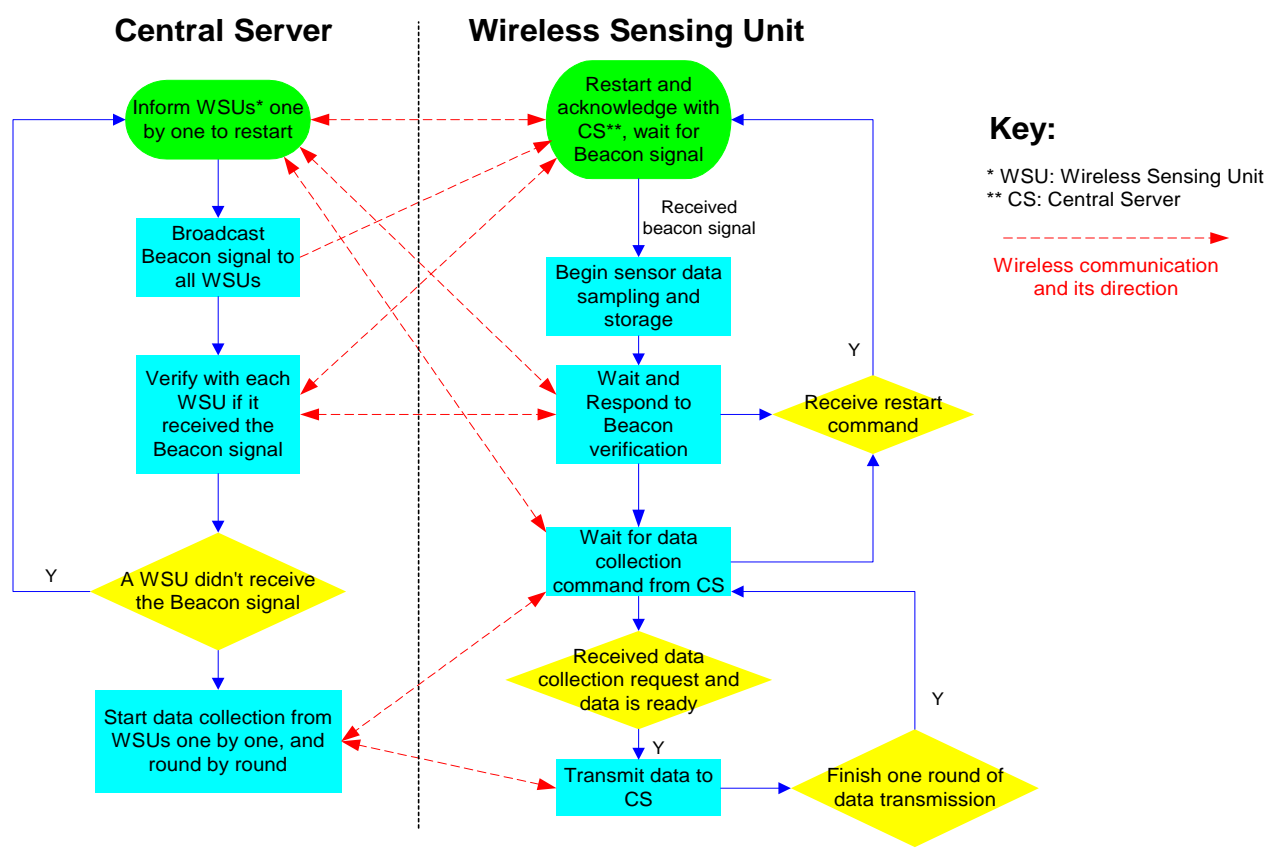

Figure 3. State diagram detailing the procedure for synchronizing the wireless sensing units 
error might potentially increase after certain periods of long time durations because of the natural drift present in the crystal clocks integrated with each wireless sensing unit. Therefore, resynchronization of the system is recommended after long periods of inactivity in the wireless sensor network.

To account for the scenario where a wireless sensing unit might not receive the beacon signal, it is necessary for all of the wireless sensing units to confirm with the central server that they have received the start signal successfully. When the central server inquires with a unit whether the unit has received the beacon signal, the server may be communicating with a unit that is still waiting for the beacon signal it never received. If the unit did not receive the beacon, then the central server realizes this problem and restarts all of the wireless sensing units by broadcasting the beacon signal again. While this communication protocol might seem time consuming, it has been timed in the laboratory and its automatic execution is found to be reasonably fast.

\subsection{Data Collection}

Once the central server confirms that all of the wireless sensing units have received the latest beacon signal, the server starts inquiring the units one by one for the data they have thus far collected. Once a unit is inquired by the server for measurement data, the wireless sensing unit transmits its most recent data. After one round of data collection, the server again starts to query each wireless sensing unit in the network for the data it collected since the last inquiry. The wireless sensing unit-central server communication during data transfer is highly reliable because of a network protocol in which the central server continues to request data from a unit until it receives an acknowledgement of that request.

A unique feature of the embedded wireless sensing unit software is that it can continue to collect data from interfaced sensors in real-time as the wireless sensing unit is transmitting data to the central server. Until the wireless sensing unit is queried for its data, the data is first stored in the unit's onboard SRAM memory bank. With over $128 \mathrm{kB}$ of space available in memory, the wireless sensing unit can effectively store up to 64,000 data points (at a 16-bit resolution).

A dual stack approach to managing the SRAM memory is taken in the current wireless sensing unit embedded software. Essentially, the available memory bank is divided into four pairs of smaller memory stacks. When a wireless sensing unit receives a request by the central server to transmit data, the embedded software establishes two memory stacks dedicated to each sensor channel for storing sensor data. The first stack for each sensor channel contains all of the sensor data that was collected before the central server requested the data. Once the wireless sensing unit receives the request for data, these stacks are no longer added to. Rather, a second set of stacks are created to store the new stream of sensor data for each channel. As the second set of stacks is being filled by the sensing interface, the wireless sensing

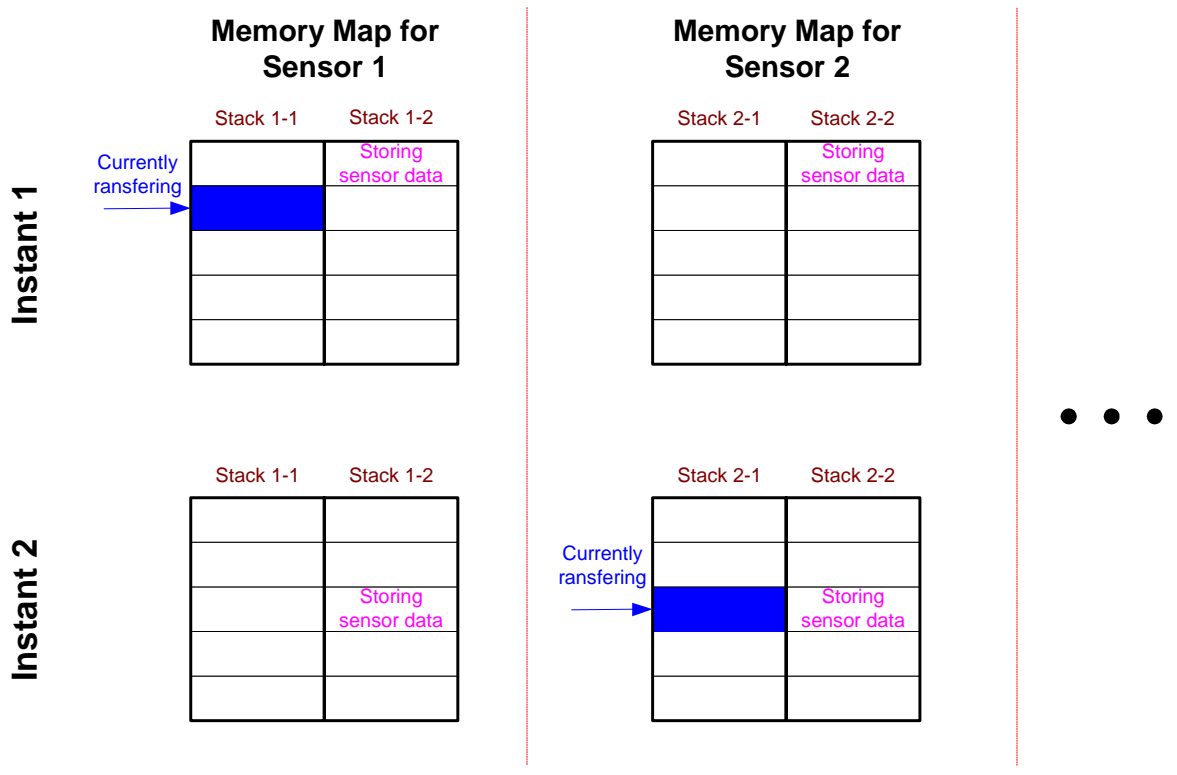

Figure 4. Dual-stack memory allocation for real-time continuous data collection 
unit is simultaneously sending the data from the first set of stacks via the wireless modem. Figure 4 illustrates the embedded operating system's management of the external SRAM memory. The two memory stacks, labeled stacks " $X$ "- 1 and " $X "-2$ for sensor " $X$ " ( $X$ is 1 or 2 in this example), are shown in the diagram for all four sensor channels. At first, the incoming data from each sensor channel is stored in stack " $X$ "-2. When the central server requests data, stack " $X$ "-2 is frozen and future data samples are written to stack " $X$ "-1. As stack " $X$ "- 1 is utilized, the wireless sensing unit is transmitting the data from the first in-first out (FIFO) stack "X"-2. It should be noted that the stacks are of a finite size so the wireless modem must be fast enough to ensure one set of stacks can be communicated to the central server before the second set of stacks fill to capacity. This is largely a function of the data rate of the 9XCite modem. In the current prototype system, the central server is able to collect raw data from up to 24 wireless sensors continuously in real-time with a sampling frequency of $50 \mathrm{~Hz}$ for each sensor. As the sample rate is raised, the number of sensors that can be utilized reduces. For example, if a sample rate of $100 \mathrm{~Hz}$ is desired, then 12 sensors can be utilized. If a high sample rate and a large number of sensors are needed, then the system is not able to simultaneously collect data and wirelessly transmit at the same time. In these instances, the wireless sensing units are first asked to collect data and only upon completion to send their data to the central server.

\subsection{Fourier Analysis using the Wireless Sensing Unit}

To test the local data processing capability of the wireless sensing unit, a fast Fourier transform (FFT) algorithm is implemented in software and embedded in the core of the wireless sensing unit. Upon demand from the central server, the wireless sensing units can be commanded to collect sensor data and to perform a floating point FFT on the sampled data. After the frequency response function is calculated, the wireless sensing unit then wirelessly communicates both the frequency response function (complex valued) and the raw time-history data. Using the currently selected microcontroller and SRAM, a 4,096-point floating point FFT takes about 18 seconds to complete. Under the wireless bandwidth limitation from 9XCite wireless modem, if a $50 \mathrm{~Hz}$ sampling frequency is applied at each sensor, and a 0 to $10 \mathrm{~Hz}$ FFT spectrum is requested to be transferred back to central server, the system can support real-time non-stopping data collection and FFT results collection from up to 11 wireless sensing units simultaneously. Depending on the specific application, it may not be necessary to transfer the original time-history data. In that case, the number of units that can support simultaneous data collection and FFT analysis may be increased. In a general sense, if the embedded local data processing algorithm is not computationally expensive and the desired results to be transmitted are small, the number of wireless sensing units that can support real-time data interrogation while simultaneously collecting sensor data can be very large.

\section{VALIDATION TESTS}

To test the performance of the proposed wireless structural monitoring system, various validation tests are performed in both the laboratory and in the field. The results obtained from both set of tests validate the wireless sensing unit is capable of accurately collecting data, performing sophisticated data interrogation, and achieving reliable wireless communication.

\subsection{Laboratory Tests}

In the laboratory tests, the proposed wireless structural monitoring system is employed to monitor the dynamic behavior of a simple 3-story test structure. The test structure is constructed of aluminum and is mounted to a shaking table in the laboratory. The reader is referred to a previous paper in which the details of the structure and the results of shake table tests are presented ${ }^{13}$. The intention of the test is to measure the stead-state acceleration response of the structure under harmonic loading and to use the wireless sensing unit to calculate the frequency response function of the acceleration response using the embedded FFT algorithm. Provided only the steady state behavior of the structure is recorded, the frequency response function should contain energy only in the harmonic frequency of the shaking table.

The shaking table is commanded to apply a harmonic base motion at a frequency of $5.7 \mathrm{~Hz}$. The selected frequency, $5.7 \mathrm{~Hz}$, is close to the second natural frequency of the lumped-mass shear-frame structure. First, the three wireless sensing units installed in the structure at each floor are commanded to collect 4096 data points at a sample rate of 100 Hz. After collecting the acceleration response of the structure, the wireless sensing units then calculate a 4096-point frequency response function using the acceleration response time-history of the structure. After the FFT computation, both the time history data and FFT results are transferred back to the central server for validation. Figure 5 illustrates 

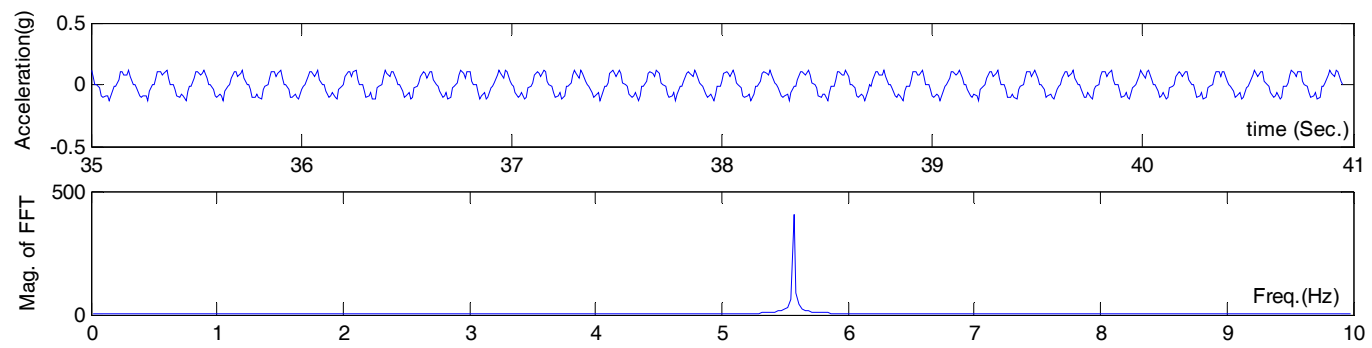

(a) Harmonic ground motion acceleration
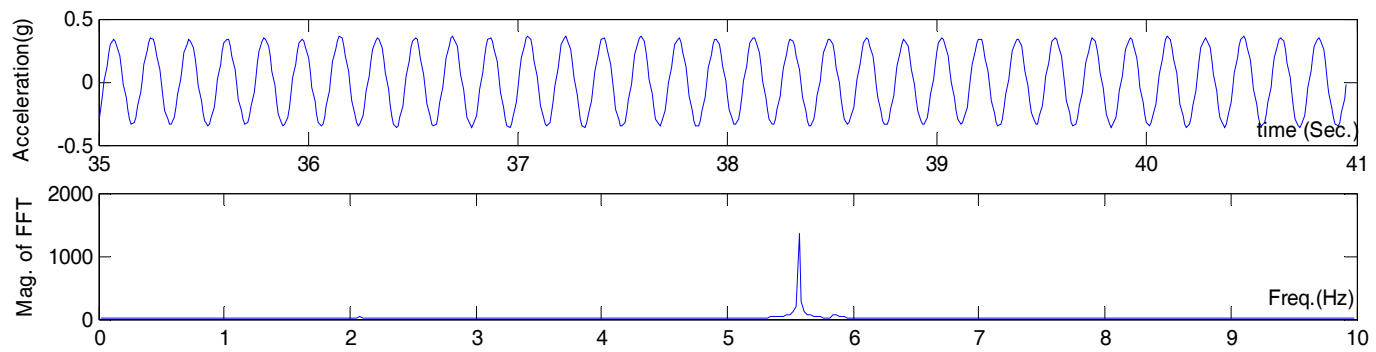

(b) Acceleration response of the third floor

Figure 5. Acceleration time history and frequency response function communicated by the wireless sensing units at the ground and third floor of the test structure

the acceleration time history and FFT results computed by the wireless sensing units at the ground and third floor for the $5.7 \mathrm{~Hz}$ harmonic base excitation. Based on the frequency response functions calculated by the wireless sensing units, the magnitude of the frequency response functions for the ground and third floor accelerations have peak values at the frequency of $5.7 \mathrm{~Hz}$.

\subsection{Geumdang Bridge Tests}

To test the performance of a large-scale wireless sensor network in the challenging environment posed by a real civil structure, field tests of the wireless structural monitoring system are conducted. The Geumdang Bridge, a long-span concrete box girder bridge spanning $122 \mathrm{~m}$, is instrumented with accelerometers attached to both a tethered and wireless monitoring system ${ }^{14}$. The depth of the box girder is $2.6 \mathrm{~m}$ while the top bridge deck has a total width of $12.6 \mathrm{~m}$. The total span is supported along its length by three concrete piers and one concrete abutment. The locations of the piers along the span length are delineated in Figure 6.

Prior to installation of the wireless monitoring system, a wire-based structural monitoring has been previously installed. The tethered monitoring system employs piezoelectric accelerometers to measure the vertical acceleration response of the bridge at the locations \#1, 3, 4, 5, 6, 7, 8, 9, 10, 11, 13, 14, 15 and 16, as denoted in Figure 6 (a). The piezoelectric accelerometers used by the wire-based monitoring system are PCB393 accelerometers manufactured by PCB Piezotronics. For direct comparison, the wireless monitoring system also deploys accelerometers at these locations, with one accelerometer installed side-by-side to each PCB 393 accelerometer. However, to reduce the cost of the wireless monitoring system, one capacitive Piezotronics PCB3801 accelerometer is attached to a wireless sensing unit at the 14 sensor locations. In total, 14 wireless sensing units are installed within the interior spaces of the box girder. Table 3 provides a side-by-side comparison of the two accelerometers installed upon the Geumdang Bridge. As illustrated in the table, PCB 393 accelerometers used by the wire-based system have higher sensitivity and lower noise. Therefore, they are expected to have better performance than the PCB 3801 accelerometers used by the wireless system. While the interior of the box girder protects the wireless sensing units from the natural elements, there are a number of vertical stiffener diaphragms within the box girder that will attenuate the wireless signal between the wireless sensing units. The central server (laptop) is installed in the vicinity of sensor location \#9, with a maximum distance between the central server and the furthest wireless sensing unit of about $60 \mathrm{~m}$. As opposed to the tethered monitoring system, 


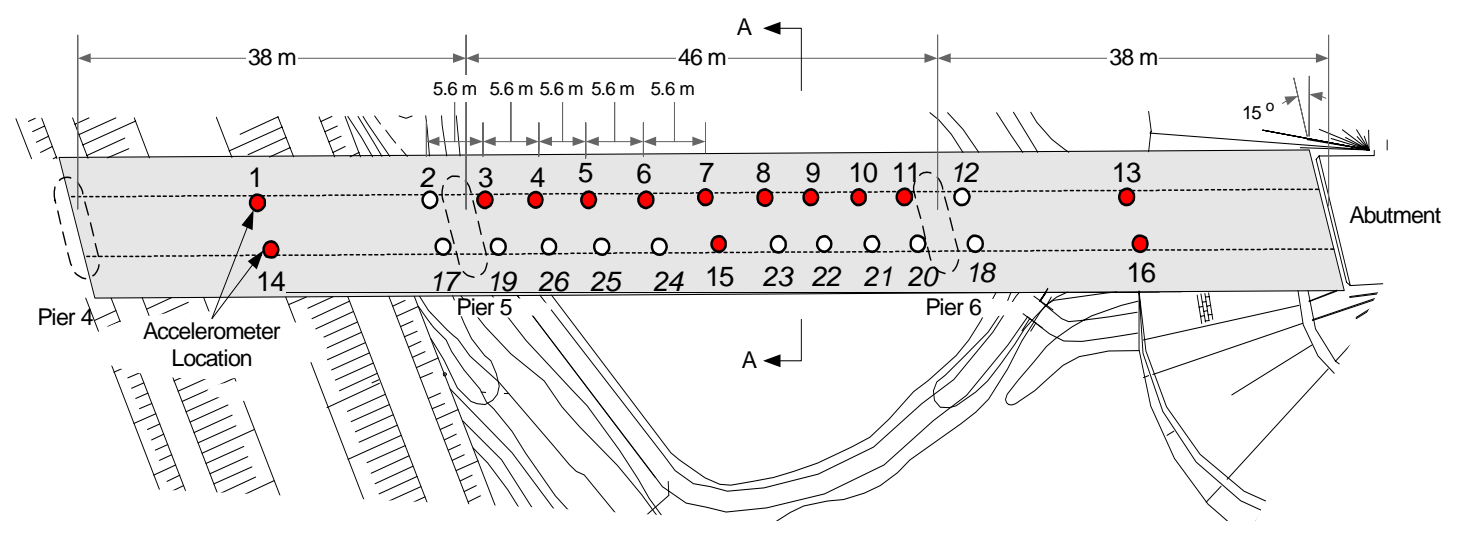

(a) Plan view

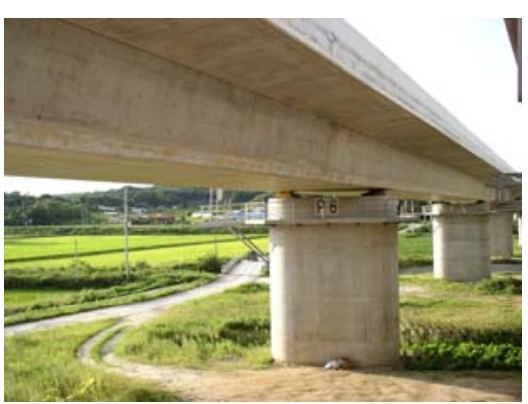

(b) Outside perspective view of the bridge

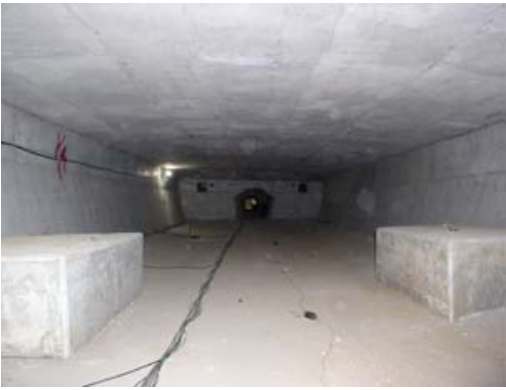

(c) Box girder interior

Figure 6. Concrete box girder Geumdang Bridge, South Korea

installation of the wireless monitoring system takes only about one hour. In later tests, the wireless sensing units are moved to different locations inside the box girder; a convenience made possible by using wireless communications.

For the tethered monitoring system, the analog outputs of PCB393 accelerometers are fed into a 16-channel PCB Piezotronics 481A03 signal conditioning unit. Before being sampled and digitized, the signals are amplified by a factor of 10 using an amplification circuit native to the signal conditioning unit. A National Instruments' 12-bit data acquisition card (model number 6062E) is used by a standard laptop computer to sample and digitize the amplified accelerometer signals. The tethered monitoring system is configured to sample the 14 sensor channels at $200 \mathrm{~Hz}$. For the wireless monitoring system, the PCB 3801 accelerometers are connected to the sensing interface of each wireless sensing unit. Due to the limited wireless communication bandwidth and the large number of wireless sensing units that are streaming data simultaneously, the sampling rate of the wireless monitoring system is selected at $70 \mathrm{~Hz}$. Over the course of two full days of testing, the wireless monitoring system never experiences any communication problems including data loses. The designed communication protocol for near-synchronized and non-stop real-time data acquisition proves to be highly reliable for the large-scale wireless sensor deployment.

Table 3. Performance comparison between accelerometers used by the wire-based system and the wireless system

\begin{tabular}{ccc}
\hline Sensor Property & PCB 393 (Wire-based System) & PCB 3801 (Wireless System) \\
\hline Sensor Type & Piezoelectric & Capacitive \\
Maximum Range & $\pm 0.5 \mathrm{~g}$ & $\pm 3 \mathrm{~g}$ \\
Sensitivity & $10 \mathrm{~V} / \mathrm{g}$ & $0.7 \mathrm{~V} / \mathrm{g}$ \\
Bandwidth & $2000 \mathrm{~Hz}$ & $80 \mathrm{~Hz}$ \\
RMS Resolution (Noise Floor) & $50 \mu \mathrm{g}$ & $0.5 \mathrm{mg}$ \\
Excitation Voltage & $18 \mathrm{VDC}$ min. & $5 \mathrm{VDC} \mathrm{min}$. \\
\hline
\end{tabular}



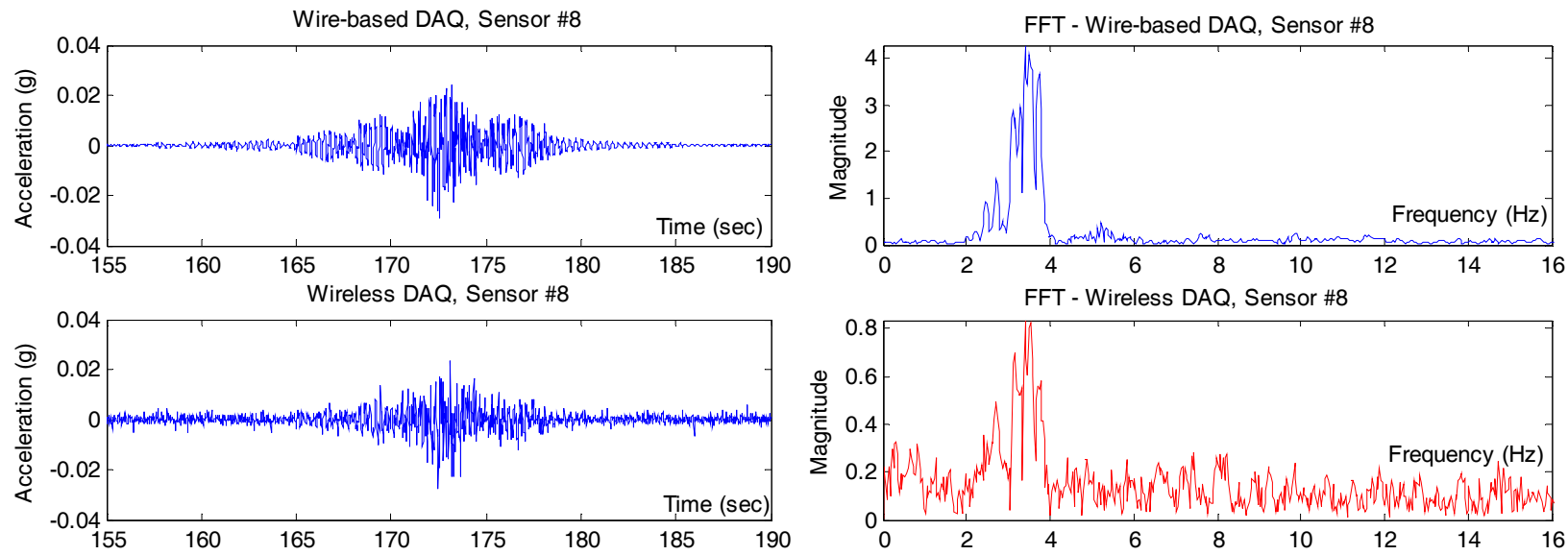

Figure 7. (Left) acceleration time-history response of the Geumdang Bridge under controlled traffic loading at sensor location \#8. (Right) corresponding frequency response functions using the measured acceleration response shown on the left.

The Geumdang Bridge is kept closed to regular highway traffic while the bridge is excited using trucks of known weight and speed crossing the bridge. Figure 7 illustrates the acceleration response of the bridge at sensor location \#8 when a 40-ton truck crosses the bridge at $60 \mathrm{~km} / \mathrm{hr}$. The figure plots the accelerometer time histories collected by the two different systems. There exists a strong one-to-one correspondence in the acceleration response time history records collected by the two systems. As expected, the acceleration record measured by the wireless monitoring system appears noisier than that collected by the tethered monitoring system. The wireless sensing units are also commanded to perform a 4096 point FFT using the measured acceleration response. The frequency response function of the Geumdang Bridge as calculated by the wireless monitoring system is shown in Figure 7. If the frequency response function is compared to the frequency response function calculated off-line using the tethered monitoring system's response data, again the primary response frequency of the bridge can be identified in both functions $(3.6 \mathrm{~Hz})$. Furthermore, the FFT results from the two systems are very close to each other. The difference in the amplitude of FFT results is mainly caused by the different sampling frequencies used in the two systems.

\section{CONCLUSION}

The integrated software and hardware architecture of a newly proposed wireless structural health monitoring system is presented in detail in this paper. Both laboratory and field validation tests corroborate the capability and reliability of the prototype system for large-scale deployment in real civil structures. Some of the special features of the prototype wireless monitoring system includes: 1) ultra-low power consumption without sacrificing long-range communication, 2) rapid system installation and low system costs, 3) reliable communication protocols ensuring lossless wireless communications, 4) embedment of a multi-threaded operating system allowing for simultaneous data collection and wireless communications, 5) high-precision time synchronization, and 6) considerable local data processing capabilities integrated with each wireless sensing unit.

There are a number of aspects of the prototype system that are yet to be improved. With respect to the wireless sensing unit hardware design, sensor signal conditioning and anti-noise filters can be designed to improve the measurement fidelity of the wireless sensing units. With the rapid development in wireless communication technologies, wireless modems that support longer communication ranges and higher data rates while consuming less power should be pursued. Improvements can also be made with respect to the embedded software. For example, more advanced communication protocols are needed to organize very-large-scale wireless sensor networks with true peer-to-peer connections. For decentralized damage detection and system identification algorithms, reliable peer-to-peer communication between wireless sensing nodes will be needed. While the Geumdang Bridge illustrated the potential of wireless structural monitoring systems to replace tethered monitoring systems, additional studies using field structures are needed to further refine the design of the prototype system. 


\section{ACKNOWLEDGEMENT}

This research is partially funded by the National Science Foundation under grants CMS-9988909 (Stanford University) and CMS-0421180 (University of Michigan). The first author is supported by an Office of Technology Licensing Stanford Graduate Fellowship. Additional support was provided by the University of Michigan Rackham Grant and Fellowship Program. The authors would like to express their extreme gratitude to Professors Chung Bang Yun and Jin Hak Yi, as well as Mr. Chang Geun Lee, from the Korea Advanced Institute of Science and Technology (KAIST) for unfettered access to Geumdang Bridge.

\section{REFERENCES}

1. S. Chase, "The role of smart structures in managing an aging highway," http://www.tfhrc.gov/hnr20/nde/ppt/ slide001.htm, Keynote Presentation, SPIE Conference on Health Monitoring of Highway Transportation Infrastructure, New Beach, CA, USA, March 2001.

2. P. C. Chang, A. Flatau, and S. C. Liu, "Review Paper: Health Monitoring of Civil Infrastructure," Structural Health Monitoring, 2(3), 257-267.

3. B. F. Spencer, Jr., M. E. Ruiz-Sandoval, and N. Kurata, "Smart sensing technology: opportunities and challenges," http://cee.uiuc.edu/sstl/papers/Smart_Sensing_Technology.pdf, Journal of Structural Control and Health Monitoring, in press, 2005.

4. E. G. Straser, and A. S. Kiremidjian, A Modular, Wireless Damage Monitoring System for Structures, Report No. 128, John A. Blume Earthquake Engineering Center, Department of Civil and Environmental Engineering, Stanford University, Stanford, CA, USA, 1998.

5. M. Celebi, Seismic Instrumentation of Buildings (with Emphasis on Federal Buildings), Report No. 0-7460-68170 United States Geological Survey (USGS), Menlo Park, CA, USA, 2002.

6. J. P. Lynch, A. Sundararajan, K. H. Law, A. S. Kiremidjian, and E. Carryer, "Embedding damage detection algorithms in a wireless sensing unit for attainment of operational power efficiency," Smart Materials and Structures, IOP, Vol. 13, No. 4, pp. 800-810, 2004.

7. V. A. Kottapalli, A. S. Kiremidjian, J. P. Lynch, E. Carryer, T. W. Kenny, K. H. Law, and Y. Lei, "Two-tired wireless sensor network architecture for structural health monitoring,” SPIE 10th Annual International Symposium on Smart Structures and Materials, San Diego, CA, USA, March 2003.

8. L. Mastroleon, A. S. Kiremidjian, E. Carryer, and K. H. Law, "Design of a new power-efficient wireless sensor system for structural health monitoring," SPIE 9th Annual International Symposium on NDE for Health Monitoring and Diagnostics, San Diego, CA, USA, March 2004.

9. J. L. Hill, System Architecture for Wireless Sensor Networks, Ph.D. Thesis, Department of Computer Science, University of California, Berkeley, CA, 2003.

10. D. E. Culler, and W. Hong, "Wireless Sensor Networks: Introduction," Communications of the ACM, Vol. 47, No. 6, pp. 30-33, 2004.

11. E. H. Callaway Jr., Wireless Sensor Networks: Architectures and Protocols, Auerbach Publications, 2004.

12. Y. Lei, A. S. Kiremidjian, K. K. Nair, J. P. Lynch, and K. H. Law, "Time synchronization algorithms for wireless monitoring system," SPIE 10th Annual International Symposium on Smart Structures and Materials, San Diego, CA, USA, March 2003.

13. Y. Wang, J. P. Lynch, and K. H. Law, "Wireless Structural Sensors Using Reliable Communication Protocols for Data Acquisition and Interrogation," Proceedings of the 23rd International Modal Analysis Conference (IMAC XXIII), Orlando, FL, USA, February, 2005.

14. J. P. Lynch, Y. Wang, K. H. Law, J. H. Yi, C. G. Lee, and C. B. Yun, "Validation of a large-scale wireless structural monitoring system on the Geumdang Bridge", Proceedings of the 9th International Conference on Structural Safety and Reliability (ICOSSAR 2005), in press. 